\title{
Heterogeneities evolving in Earth's mantle due to melt depletion
}

\author{
LENA NOACK, ALEXANDER BALDUIN
}

Freie Universität Berlin, Institute of Gelogical Sciences, Malteserstr. 74-100, D-12249 Berlin, Germany

The local composition of Earth's mantle changes over time mainly due to melt extraction, subduction of crustal material, and inefficient mantle mixing. In the Archean, mantle temperatures exceeded present-day values by $\sim 150$ 200 degrees, which affected the phase transition from upper to lower mantle, such that material mixing between these layers may have been reduced. We investigate how unknown early Earth mantle conditions may have influenced the phase transition Clapeyron slopes and hence global mantle convection and mixing.

We find that under Archean conditions, the upper mantle becomes more and more depleted, while the lower mantle stays primordial in composition for a long time span. The reason for this evolving heterogeneity is that the lighter harzburgite material resists transportation to the lower mantle at to the ringwoodite-perovskite phase transition. A cooler mantle together with the initiation of plate tectonics can later lead to more efficient mixing of Earth's upper and lower mantle. 